Originalveröffentlichung in: Christoph Nyiri (Hrsg.), Tradition. Proceedings of an international research workshop at IFK, Vienna 10-12 June 1994, Wien 1995, S. 45-60

\title{
Fünf Wege zum Kanon. \\ Tradition und Schriftkultur im alten Israel und frühen Judentum
}

\section{Jan Assmann}

\section{Zwei Vorbemerkungen}

\subsection{Verschriftlichung}

Der Begriff der Tradition hat zwei Bedeutungen. Wenn wir ihn vom Standpunkt von Gedächtnis und Erinnerung aus betrachten, wie es z.B. Maurice Halbwachs getan hat, dann erscheint er als der Gegensatz zum Gelebten, Verkörperten und Kommunizierten und als der Inbegriff des in symbolischen Formen ausgelagerten und von Institutionen verwalteten Wissens. ${ }^{1}$ Wenn wir ihn dagegen vom Standpunkt der Schrift aus betrachten wie in der jüdischen und der katholischen Tradition, dann erscheint er als der Gegensatz des schriftlich Fixierten und der Inbegriff des an lebendige Träger gebundenen, inkarnierten Wissens. Der Begriff der Tradition changiert zwischen diesen beiden Extremen der Erinnerung und der Schrift. Er grenzt sich gegenüber der Erinnerung $\mathrm{ab}$ als das soziale, normative, wenn auch nicht unbedingt vollständig sprachlich ausformulierte Wissen, und gegenüber der Schrift als das in weiten Bereichen implizite, außersprachliche, über mimetisches Vor- und Nachmachen weitergegebene und nichtschriftliche Wissen. Zwar gehört natürlich auch Schriftliches zur Tradition, aber die Tradition geht weit darüber hinaus. Zugespitzt könnte man sagen, daß lebendige, verkörperte Erinnerung und Kommunikation ihren Tod in der Tradition finden, und die lebendige, verkörperte Tradition ihren Tod in der normativen Schriftlichkeit.

Ein Beitrag über Gedächtnis und Tradition wird die Grenze zwischen dem gelebten und dem formulierten, dem individuellen und dem kulturellen, dem impliziten und dem expliziten Wissen thematisieren. Bei diesem Beitrag über Tradition und Schriftkultur geht es dagegen um die andere Grenze: zwischen der Tradition in ihren impliziten und mündlichen Aspekten auf der einen, und der expliziten und normativen Schriftlichkeit auf der anderen Seite. So gesehen erscheinen Tradition und Schrift als Gegensätze bzw. als komplementäre Aspekte. Traditionen werden normalerweise nicht verschriftet. Geschieht das

1 M. Halbwachs, La mémoire collective, Paris 1950. 
doch, verweist das auf einen Traditionsbruch, zumindest eine Krise. Die Tendenz zur Verschriftung ist in Traditionen nicht unbedingt im Sinne einer inneren Entwicklungslogik angelegt. Der natürliche Weg der Tradition führt nicht zur Schrift, sondern zur Gewohnheit, nicht zur Explikation, sondern zum Implizit-Werden, zur Habitualisierung und Unbewußtmachung. ${ }^{2}$ Der Anstoß zur Verschriftlichung muß von außen kommen und wo er kommt, verändert er die Tradition. Daher ist es sinnvoll, nach solchen äußeren Anstößen der Verschriftung zu fragen. Ich will das am Beispiel Israels versuchen und fünf verschiedene solcher Anstöße namhaft machen, die hier nicht nur zur Verschriftung, sondern über die Verschriftung hinaus zur Kanonisierung der Tradition geführt haben.

\subsection{Kanonisierung}

Kanonisierung ist eine besondere Form von Verschriftlichung. Die Texte werden nicht einfach niedergeschrieben, sondern in ihrer Verbindlichkeit gesteigert. Diese gesteigerte Verbindlichkeit bezieht sich auf ihre Gestalt (ihren Wortlaut), sowie auf ihre Autorität, was eng miteinander zusammenhängt. Autorität bedeutet, daß alles, was der Text sagt, schlechthin normative Geltung besitzt und daß alles, was normative Geltung beansprucht, sich als Sinn dieses Textes muß ausweisen können. Damit ist zugleich gesagt, daß der Text weder fortgeschrieben noch um weitere Texte ergänzt werden kann, sondern $\mathrm{da} B$ fortan aller weiterer Sinn aus dem Text selbst gewonnen werden muß. Diese Schließung bedingt seine Gestalt, die nun in ihrem Wortlaut fixiert wird. Es handelt sich hier um eine wirkliche Endgestalt. Die Normativität des Textes, seine Autorität und Hochverbindlichkeit, bezieht sich ausschließlich auf diese Endgestalt, nicht auf irgendwelche Vor- und Urstufen. Es gibt innerhalb dieser kanonischen Endgestalt kein mehr oder weniger an Verbindlichkeit, keine wichtigeren und unwichtigeren Sätze, keine ursprünglichen und sekundären Partien. Mit der Endgestalt ist ist das geschichtliche Werden des Textes vergessen.

Der Prozeß der Kanonisierung stellt das normale Schicksal geschriebener und abgeschriebener Texte auf den Kopf. Denn der übliche Weg ist der Weg bergab: Textgeschichte ist Verfallsgeschichte.

2 Zum Prozeß der Habitualisierung vgl. P. Bourdieu, Zur Soziologie der symbolischen Formen, Frankfurt 1974, 125-158. Zum Prozeß der Unbewußtwerdung s. die Beitäge von Mario Erdheim: Die gesellschaftliche roduktion von Unbewußtheit, Frankfurt 1984 und Die Psychoanalyse und das nbewußte in der Kultur, Frankfurt 1988. 
Daher bemüht sich jede philologische Textarbeit um die Erschließung der ältesten und ursprünglichsten Fassung, des Archetyps. Die Quelle des Sinns ist die Intention des Autors, und je näher eine Fassung dieser Quelle steht, desto sinnvoller ist sie. Textkritik arbeitet sich von der Letztform zurück zur Urform, Kanonkritik arbeitet umgekehrt die Tendenzen heraus, die das Werden, Wachsen, Zusammenwachsen und Heiligwerden der Texte vorantreiben. Hier interessieren nicht die ursprünglichen Autoren und ihre Intentionen, sondern die Redaktoren und insbesondere die Letztredaktion, die das Ganze zum Kanon zusammenschließt. ${ }^{3}$ In theologischer Hinsicht kann man Kanonisierung als einen inspirierten Prozeß verstehen, als eine Offenbarung, die sich in der Länge der Zeit entfaltet und vollendet, ja, nach rabbinischer Auffassung, sich in Form der mündlichen Torah sogar noch in die Auslegung des Textes hinein fortsetzt. ${ }^{4}$ Ich möchte im Folgenden jedoch nicht als Theologe, sondern als Historiker einen Blick auf den Prozeß der Kanonisierung werfen und am Fallbeispiel Israels fünf Tendenzen oder Triebkräfte namhaft machen, die sich teilweise auch in anderen Kulturen wiederfinden lassen, teilweise aber auch ein Spezifikum der altisraelitisch-frühjüdischen Situation sind.

\section{Fünf Impulse der Kanonisierung}

\subsection{Die Exkarnation der Gesetze und die Erfindung einer normativen Vergangenheit: die josianische Situation (Kanon von unten)}

Der erste Schritt von der Tradition zur Schrift und darüberhinaus zum Kanon wird im Umkreis der Rechtskodifikation vollzogen. Im Allgemeinen sieht man darin nicht viel Anderes als die Übernahme mesopotamischer Formen der Rechtsverschriftung, die dort bis ins 3 . Jahrtausend zurückreichen. ${ }^{5}$ Das scheint mir jedoch verfehlt. In Mesopotamien haben wir es mit Rechtsbüchern zu tun. Die Torah

3 Also die Textschicht "R" (Redaktor), die M. Buber und F. Rosenzweig als Rabbenu" (unser Lehrer), also die verbindliche Lehrautorität im Sinne des "impliziten Autors" auffaßten.

4 Einer der Hauptvertreter dieser "Kanonhermeneutik" ist J. A. Sanders, vgl. seine Bücher Torah und Canon (1972), Canon and Community (1984) und From Sacred Story to Sacred Text (1987), sämtlich Fortress Press, Philadelphia. Zum Begriff der inspirierten Auslegung vgl. P. Schäfers Beitrag in J. Assmann, B. Gladigow (Hrsg.), Text und Kommentar (im Druck).

5 H. G. Kippenberg, Die vorderasiatischen Erlösungsreligionen in ihrem Zusammenhang mit der antiken Stadtherrschaft, Frankfurt 1991, 157ff. mit Hinweisen auf einschlägige Literatur. 
dagegen ist ein Gesetzbuch. Der Unterschied ist fundamental. Ich würde ihn in kurzen Worten folgendermaßen charakterisieren. ${ }^{6}$

Das Rechtsbuch ist eine Gattung der bürokratischen Wissensliteratur, die in Mesopotamien bekanntlich in einzigartiger Weise entwickelt ist. Das Rechtsbuch ist aber kein Kodex, d.h. kein Gesetzbuch: es hat keinen präskriptiven, absolut bindenden Charakter. Wir haben es hier mit zwei ganz verschiedenen Funktionen von Schrift zu tun. Die eine Funktion der Schrift ist die der Speicherung, im Sinne einer Extension und Exteriorisierung des Gedächtnisses ${ }^{7}$, die andere ist die der verbindlichen Veröffentlichung, im Sinne einer Extension und "Exkarnation" - diesen Begriff übernehme ich von Aleida Assmann ${ }^{8}$ des höchstrichterlichen (legislativen oder iudicativen) Machtworts. Im einen Fall unterstützt die Schrift das Gedächtnis, im anderen die Stimme. Im einen Fall wird ein Gesetz aufgeschrieben, weil es gilt (und überdauert als Schrifttext die Zeit seiner Geltung, während sonst mit der Geltung auch die Erinnerung daran verschwinden würde), im anderen Fall gilt ein Gesetz, weil es geschrieben steht. Die eine Funktion könnte man "informativ" nennen; sie dient zur Sicherung und Vermittlung relevanten juristischen Wissens. Die andere Funktion wäre demgegenüber als "performativ" zu bezeichnen: hier wird wird mit den Mitteln der Schrift eine sprachliche Handlung vollzogen. ${ }^{9}$ Ein Gesetzbuch als Kodifikation geltenden Rechts gehört zur performativen Schriftlichkeit: es stellt den Sachverhalt einer Rechtsordnung her, den sie beschreibt. Das Rechtsbuch dagegen stellt die für die Formulierung von Gesetzen und Urteilen notwendige Wissenstradition bereit, aber gibt diese Gesetze und Urteile nicht in verbindlicher Weise vor. Es ist eine Hilfe, aber keine Vorschrift bei der Rechtsfindung. Die Legitimität der Gesetze entspringt nicht einer kodifizierten Tradition, sondern der jeweiligen königlichen Autorität. Die Gesetze müssen immer neu vom König in Kraft gesetzt oder verändert werden. Die Schriftlichkeit allein sichert ihnen weder Legitimität noch Autorität.

6 Zum Folgenden vgl. H. J. Gehrke (Hrsg.), Verschriftlichung sozialer Normen und Kodifizierung von Recht im interkulturellen Vergleich (erscheint als Band der Reihe ScriptOralia, Tübingen).

7 Vgl. hierzu v.a. A. Leroi-Gourhan, Le geste et la parole Bd. 2, La mémoire et les rhythmes, Paris 1965.

8 Aleida Assmann, "Exkarnation: Über die Grenze zwischen Körper und Schrift", in: Alois M. Müller, J. Huber (Hrsg.), Interventionen, Basel 1993, 159-181.

9 Zum Phänomen performativer Schriftlichkeit vgl. Verf., "Inscriptional Violence and the Art of Cursing: A Study of Performative Writing", in: Stanford Literature Review 9, 1992, 43-65 (deutsch in: H.U. Gumbrecht, K.L. Pfeiffer [Hrsg.], Schrift, München 1993, 233-256). 
Wo es einen König gibt, zu dessen Hauptaufgaben es gehört, Gesetze zu erlassen und in Kraft zu setzen, braucht man kein Gesetzbuch; im Gegenteil: das würde die legislative Kompetenz des Königs in ungebührlicher Weise einschränken. Das Gesetzbuch ersetzt daher in gewisser Weise den König. Und genau dies ist der Punkt. Die Torah tritt an die Stelle des altorientalischen Rechtskönigtums. Sie verschriftet nicht das juristische Wissen, sondern das königliche Machtwort, das aufgrund dieses autoritativen Anspruchs als Wort Gottes kodifiziert wird. Dadurch wird man von einem Königtum unabhängig. Dieser Schritt hat in den altorientalischen Schriftkulturen aus guten Gründen keine Parallele, wohl aber in einigen Stadtstaaten des archaischen Griechenlands, vor allem in Kreta sowie in unteritalischen Kolonien. ${ }^{10}$ Ich fasse diese Prozesse der Gesetzeskodifizierung unter dem Stichwort der josianischen Situation zusammen, aber weiß natürlich, daß sie sich über eine weitaus längere Epoche der israelitischen Geschichte erstrecken. ${ }^{11}$ Die Regierungszeit des Joschija bedeutet hier lediglich einen Durchbruch und Höhepunkt. Hier werden die Gesetze und normativen Traditionen nicht nur gesammelt, sondern umfassend kodifiziert und in Kraft gesetzt, und zwar mit erheblicher revolutionärer Gewalt. ${ }^{12}$ Die josianische Reform hat nicht nur kultischen, sondern auch politischen Charakter: den Charakter einer revolutionären nationalen Erweckungsbewegung in der für solche Bewegungen typischen Semantik der Erinnerung an ein vergessenes Eigenes und der sich aus solcher Erinnerung speisenden Befreiung vom und Vertreibung des Fremden.

\subsection{Die Exkarnation der Tradition: die babylonische Situation}

Wenn wir hier den von Aleida Assmann eingeführten Begriff der' Exkamation ${ }^{13}$ auf "Tradition" anwenden, dann verstehen wir unter Tradition das gelebte, in Trägern verkörperte und in lebendigem Umgang sowohl durch sprachliche Unterweisung als auch und vor allem durch sprachloses Vormachen und Nachmachen weitergegebene und in weiten Bereichen selbstverständlich, unbewußt und implizit gewordene Wissen. Die typische Situation, in der solches Wissen aus der impliziten Sprachlosigkeit und der mündlichen Unterweisung herausgeholt und verschriftet wird, ist der Traditionsbruch, wenn die

10 Vgl. hierzu K.J.Hülkeskamp, "Written Law in Archaic Greece", in: Proceedings of the Cambridge Philolocical Society 38, 1992, 87-117.

11 Vgl. hierzu F. Crüsemann, Die Tora. Theologie und Sozialgeschichte des alttestamentlichen Gesetzes, München 1992.

12 Crüsemann, a.a.O., 235-322.

13 Vgl. Anm. 8. 
Kette des Vormachens und Nachmachens sowie der mündlichen Kommunikation abreißt. In solchen Situationen entstehen nicht nur Texte, sondern erhalten vor allem schon vorhandene Texte erhöhte Normativität. Wo der Kontakt mit den lebendigen Vorbildern abreißt, beugt man sich über die Texte auf der Suche nach Orientierung. Das ist die Situation der babylonischen Gefangenschaft und der Diaspora. Viele Spielarten dessen, was heute als Fundamentalismus verbucht wird, erwuchsen aus den Traditionsbrüchen des zweiten Weltkriegs, des Holocaust und der Kolonisierung.

Das Deuteronomium beschreibt und kodifiziert diesen Übergang aus der gelebten in die gelernte Tradition als Übergang von der Augenzeugenschaft und lebendigen Erinnerung der Wüstengeneration in das kulturelle Gedächtnis Israels, das auf eine äußerst elaborierte Mnemotechnik gegründet wird. In diesem Übergang konstituiert sich Israel als Lern- und Erinnerungsgemeinschaft. ${ }^{14}$ Dieser bis heute absolut zentrale Aspekt der jüdischen Identität konstituiert sich, wie die deuteronomistischen Texte deutlich belegen, bereits in der Situation des babylonischen Exils. Hier wird die Religion von einer Sache kultischer Reinheit zu einer Sache des Lernens und der Bildung. Dem priesterschriftlichen Ideal des goj qadosch, des "heiligen Volks" von Priestern ${ }^{15}$, entspricht das deuteronomistische Ideal des "weisen und gebildeten Volkes" (cam Hakham wenavon) von dem in Dtn 4.6 die Rede ist. Das weise und gebildete Volk hat die verschriftete Torah gelernt, und zwar auswendig gelernt. ${ }^{16}$

Die verschriftete Tradition kann nicht mehr einfach nachgelebt, sie muß gelernt werden. Mit der Deportation ins babylonische Exil verschwindet das selbstverständliche Vorbild der älteren Generationen. Die normative Tradition muß verschriftet werden, weil sie nicht mehr

14 Vgl. hierzu Georg Braulik OSB, "Das Deuteronomium und die Gedächtniskultur Israels. Redaktionsgeschichtliche Beobachtungen zur Verwendung von Iamad", in: G. Braulik, W. Groá, S. McEvenue (Hgg.), Biblische Theologie und gesellschaftlicher Wandel (Fs. Norbert Lohfink SJ), Freiburg 1993, 9-31, im Anschluß an N. Lohfink, "Der Glaube und die nächste Generation. Das Gottesvolk der Bibel als Lerngemeinschaft", in: N.L., Das Jüdische am Christentum, Freiburg 1987, 144-166 und Jan Assmann, "Religion als Erinnerung: das Deuteronomium als Paradigma kultureller Mnemotechnik", in: Das kulturelle Gedächtnis, 212-228. we-'atüm tahju-li mamlükhüt kohanim wegoj qadosch Ex 19.6

16 Ähnliches gilt übrigens auch, einem Hinweis von H.J. Gehrke zufolge, für die Gesetzeswerke im archaischen Kreta, die nach einer Notiz bei Aelian in Verbindung mit Melodien auswendig gelernt wurden. Der musische Lernstoff bestand an erster Stelle aus den Gesetzen, dann kamen Götterhymnen und zuletzt Loblieder auf verdiente Vorfahren. Der Doppelsinn des griechischen Wortes nomos "Gesetz" und "Lied" (davon "Neumen" für musikalische Notation) leitet sich von dieser Mnemotechnik ab. 
einfach nachgemacht werden kann. Die Normativität der Texte muß geprüft und festgelegt werden, um etwas zu haben, woran man sich halten kann. "In Augenblicken sozialer Desorganisation", schreibt Helmut Lethen mit Bezug auf die Situation zwischen erstem und zweiten Weltkrieg, "in denen die Gehäuse der Tradition zerfallen und Moral an Überzeugungskraft einbüßt, werden Verhaltenslehren gebraucht, die eigenes und Fremdes, Innen und Außen unterscheiden helfen. Sie ermöglichen, Vertrauenszonen von Gebieten des Mißtrauens abzugrenzen und Identität $\mathrm{zu}$ bestimmen". ${ }^{17}$ Traditionsbrüche bedeuten Verschriftungsschübe. Das war im Ägypten des ausgehenden 3.Jahrtausends, nach dem Untergang des Alten Reichs, nicht anders. Die erste Zwischenzeit bedeutete nicht nur einen Traditionsbruch, sondern auch einen Verschriftungsschub, der sich in allererster Linie auf die Normen des sozialen Lebens bezog. Der zerfallene Vertrauenshorizont mußte im Raum der Schrift explizit kolonisiert und abgegrenzt werden. ${ }^{18}$

\subsection{Die Kodifizierung der normativen Überlieferung: die persische Situation (Kanon von oben)}

Das Perserreich befestigte seine Herrschaft in den Provinzen, indem es sich zum besonderen Anwalt und Hüter der lokalen Tradition, der "patrioi nomoi" machte. ${ }^{19}$ In Ägypten wurde eine Kommission eingesetzt, die das frühere, bis zum 44.Lebensjahr des Amasis gültig gewesene Recht erfassen sollte ${ }^{20}$. Ein gewisser Udjahorresne wurde beauftragt, die Lebenshäuser, die den Tempeln angeschlossenen Skriptorien wiederherzustellen, die die wichtigsten Überlieferungsinstitutionen waren (A.B.Lloyd 1982a). Der Tempel, den Darius I. in el-Khargeh errichten lie $B$, kann als der erste Vertreter des neuen Tempeltyps gelten, dessen Dekoration sich nicht nur auf das

17 Helmut Lethen, Verhaltenlehren der Kälte. Lebensversuche zwischen den Kriegen, Frankfurt 1994, 7.

18 Vgl. hierzu meinen Beitrag in H.J. Gehrke (Hg.), Verschriftung sozialer Normen.

19 Dieses Verfahren formuliert Hans G. Kippenberg als ein allgemeines Prinzip imperialistischer Politik: "Wenn Kolonisatoren aus den von ihnen eroberten Territorien ein Imperium machen wollen, dann müssen sie sich zu Beschützern oder geradezu zu Erfindern der Traditionen der unterworfenen Ethnien machen", in: H.G. Kippenberg, "Die jüdischen Überlieferungen als patrioi nomoi", in: R. Faber, R. Schlesier (Hrsg.), Die Restauration der Götter. Antike Religion und NeoPaganismus, Würzburg 1986, 45-60, hier S. 51, mit Verweis auf J.H. Grevemeyer (Hg.), Traditionale Gesellschaften und europäischer Kolonialismus, München 1973. Vgl. auch P. Frei/K. Koch, Reichsidee und Reichsorganisation im Perserreich, OBO 55, Fribourg 1984.

20 W. Spiegelberg, Die sogenannte Demotische Chronik (DemSt7), Leipzig 1914, 30-32; E. Meyer, Agyptische Dokumente aus der Perserzeit, SPAW 1915, 304ff. 
Kultgeschehen bezieht, sondern auch wichtige Wissensbestände aufzeichnet und den wir in Ägypten als den symbolischen Ausdruck einer Umzäunung und Verfestigung von Überlieferung und Identität bezeichneten. ${ }^{21}$ Die persische Herrschaft bedeutet für Ägypten eine Repristinierung und Kodifizierung der Traditionen.

Das jüdische Gegenstück zum Ägypter Udjahorresne ist Esra, der Schreiber, zu dem gesagt wird:

"Denn du bist von dem König und den sieben Räten ausgesandt und sollst nach dem Gesetz deines Gottes, das in deiner Hand ist, untersuchen, wie es in Juda und Jerusalem steht."22

Mit Esra und seinem Gesetzbuch ${ }^{23}$ erreicht die Verschriftung der normativen Traditionen Israels eine weitere Stufe in Richtung auf den Kanon der hebräischen Bibel. Am Wassertor von Jerusalem, an dem Esra, wie Nehemia im 8.Kap. berichtet, die gesamte Torah vor allem Volk nicht nur vorlesen, sondern zugleich auch Abschnitt für Abschnitt auslegen ließ, schlägt, wie der jüdische Historiker Y.H.Yerushalmi sich ausdrückt, nicht nur die Geburtsstunde der Schrift, sondern auch die der Exegese. "Und Esra schlug das Buch vor den Augen des ganzen Volkes auf..., und als er es aufschlug, erhob sich das ganze Volk. (...) So las man denn aus dem Buche des Gesetzes Abschnitt für Abschnitt und erläuterte es, sodaß die Leute auf die Vorlesung achthatten" (Neh $8,5 ; 8)$. Heiliger Text und Gesetzbuch sind hier zum ersten Mal eins geworden. "Zum ersten Mal in der Geschichte", schreibt Yerushalmi, "hört ein heiliger Text auf, das exklusive Gut der Priester zu sein und wird Gemeinbesitz des Volkes. Hier haben wir die Geburtsstunde der Schrift und zugleich die Geburtsstunde der Exegese."24

So hatte schon Josia im Jahre 622 das im Tempel zufällig aufgefundene sefer ha-berit oder sefer ha-torah vor allem Volk verlesen lassen. Aber damals war die Situation anders. Es handelte sich um eine Revolution. Die Schrift sollte nicht einen status quo legitimieren, sondern im Gegenteil einen schwersten Traditionsbruch, eine Kulturrevolution von ungeheurer Radikalität und Härte. Allerdings ist auch die neuerliche Inkraftsetzung des Gesetzes unter Esra nicht frei von Gewalt, wovon

21 Vgl. hierzu "Der Spätzeittempel als Kanon", in: Das kulturelle Gedächtnis, 177195.

22 Esra 7,14.

23 Esras persischer Titel lautete "Schreiber des Gesetzes des Himmelgottes"; H.H. Schaeder, Ezra der Schreiber 1930 wollte in Esra einen persichen Staatssekretär und Sonderbeauftragten für jüdische Angelegenheiten sehen.

24 Y. H. Yerushalmi, "Reflexions sur l'oubli", in: Usages de l'oubli, Colloques de Royaumont, Paris 1988, 7-21, hier S.15. 
der Bericht von der Auflösung der Mischehen zeugt. Aber die Figur des Königs ist verschwunden und die Restitution des Gesetzes vollzieht sich in einem weitgehend entpolitisierten Raum. Das "Gesetzbuch" wurde jetzt zum Kanon ausgebaut. Vorbedingung des Kanons ist das Ende der Prophetie. ${ }^{25}$ Prophetie hat in dem entpolitisierten Raum der Provinz Jehud keinen Ort mehr, die ein Teil der Satrapie Babylonien geworden ist. Die Propheten reden im Auftrage Jahwes zu König und Volk; jetzt ist schon der Satrap weit weg, wieviel weiter der König. An die Stelle des Propheten tritt der Schriftgelehrte, der die Überlieferung kodifiziert, kanonisiert und auslegt.

Den entpolitisierten Charakter des geistigen Klimas, in dem diese Arbeit vor sich ging, bezeugt vor allem die als "Priesterschrift" bekannte Komponente der Tradition. Die Vorstellungen vom legitimen Königtum und die messianischen Erwartungen, die in den prophetischen und deuteronomistischen Traditionen eine so zentrale Rolle spielen, sind hier so gut wie ganz ausgeblendet. Die monotheistische Religion der Jahwe-allein-Bewegung, die sich vor dem Exil als eine Gegen-Kultur formiert hatte ${ }^{26}$, wird nun zur "Binnenkultur" im Großverband des persischen Reiches, die sich um die Reinheit des Lebens, der Lehre und der Auslegung kümmert und die In-Gang-Haltung der weltlichen Geschäfte an die persische Besatzungsmacht delegieren kann.

Der Prozeß einer Entpolitisierung des öffentlichen Lebens beginnt sich in der Perserzeit allgemein durchzusetzen. In Ägypten und Babylonien beobachten wir die "Klerikalisierung" der Kultur, den Übergang vom Schreiber-Beamten zum Schreiber-Priester als dem repräsentativen Kulturträger, in Israel den Übergang vom Propheten zum Schriftgelehrten. Aber nur in Israel hatte sich die Religion zu einer wirklichen Alternative kollektiver Identitätsfundierung verfestigt und ausdifferenziert. Nur hier war ein "Volk" entstanden, das seine

25 J. Blenkinsopp, Prophecy and Canon. Notre Dame 1977; B. Lang, "Vom Propheten zum Schriftgelehrten. Charismatische Autorität im Frühjudentum", in: H. v. Stietencron, Theologen und Theologien in verschiedenen Kulturkreisen, Düsseldorf 1986, 89-114; vgl. auch S.Z. Leiman, The Canonization of Hebrew Scripture: The Talmudic and Midrashic Evidence, Hamden 1976. Zum Ende der Prophetie vgl. Josephus Flavius, Contra Apionem I 38-41. Die Geschichte von Moses bis Artaxerxes schrieben die Propheten in 13 Büchern. Die restlichen 4 Bücher enthalten Hymnen an Gott und Vorschriften für die Führung des menschlichen Lebens. Von Artaxerxes bis in unsere Zeit exisitert eine Überlieferung, die aber nicht gleiche Wertschätzung genießt, weil die Folge der Propheten abriß. Nur was diese uns hinterließen, verehren wir als unsere Schriften.

26 B. Lang, "The Yahweh-Alone Movement and the Making of Jewish Monotheism", in: Monotheism and the Prophetic Minority, Sheffield 1983, 13-59. 
Abgrenzung nach außen und seinen Zusammenschluß nach innen vollkommen unabhängig von politischen und territorialen Bindungen definierte, nämlich allein durch die Bindung an "das Gesetz und die Propheten". ${ }^{27}$

\subsection{Der Zaun um die Wahrheit: die hellenistische Situation \\ Die Entstehung von textual communities im Sinne von Brian Stock. \\ Dissidence und Textbesitz.}

Brian Stock hat gezeigt, daß die häretischen Bewegungen des Mittelalters sich auf hochverbindliche Texte stützten, deren Bestand und/oder deren Interpretation ihnen eigen war. Sie konnten ihren Bruch mit der offiziellen Tradition und ihren Sonderweg nur legitimieren, indem sie auf einen Text verweisen konnten, dessen Autorität und normative Ansprüche als allen traditionellen und institutionellen Ansprüchen übergeordnet dargestellt werden konnte. Dissidenz setzt Literalität voraus. Brian Stock hat daher auf diesen Typ von Bewegungen den Begriff der "Textgemeinschaft" geprägt (textual communities). ${ }^{28}$ Viele der von ihm herausgestellten Kennzeichen dieser Bewegungen des 11. und 12.Jhs. gelten bereits für die Gemeinde von Qumran und für zahlreiche ähnliche Gruppierungen wie Orphiker, Pythagoräer, Gnostiker, Urchristen, Hermetiker usw., die sich im Hellenismus und in der Spätantike typischerweise auf der Basis eines Grundbestands normativer Literatur zusammenschlossen. ${ }^{29}$ Kennzeichen einer textual community ist einerseits die identitätsdefinierende Bedeutung eines solchen Grundtexts, zum anderen die Struktur von Autorität und Führerschaft, die sich aus der Kompetenz im Umgang mit Texten ergibt. Philologische und politische Kompetenz fallen hier zusammen. Die Führung gebührt dem, der die umfassendste Kenntnis und die einleuchtendste Deutung der Texte besitzt.

27 Abgrenzung nach außen: nicht durch territoriale Grenzen, sondern durch die limitische Symbolik der Lebensform und Handlungsweise, v.a. durch Gesetze, die den Kontakt mit Nichtmitgliedern erschweren (Sabbatruhe, Exogamieverbot, Kommensalitätsverbot usw.); Zusammenschluß nach innen: durch Betonung der Mitgliedschaft, wie sie aus der Fülle der neuen Selbstbezeichungen spricht, z.B. "die Kinder des Exils" (bene haggolah), "der Rest", "die Männer des Bundes", "die Versammlung" (qahal), "die Gemeinde" (jahad), synagogé usw. Vgl. E.P. Sanders, Jewish and Christian Self-Definition II, Philadelphia 1981.

28 Brian Stock, "Textual Communities", in: The Implications of Literacy. Written Language and Models of Interpretation in the Eleventh and Twelfth Centuries, Princeton 1983, 88-240.

29 Vgl. hierzu E.P. Sanders, Jewish and Christian Self-Definition, Philadelphia 1980, 1981 und 1984. 
Die Funde von Qumran und Nag Hammadi geben uns Einblicke in die Überreste solcher Bibliotheken, auf die sich Textgemeinschaften der Antike stützten. Trotz ihres fragmentarischen Zustands lassen sie darauf schließen, daß solche Bibliotheken nicht wie heutige Bibliotheken auf möglichste Fülle und Vielfalt angelegt waren, sondern sich auf die für die Gemeinschaft verbindliche Literatur beschränkten. Diesen Charakter einer Hand- und Arbeitsbibliothek haben sie mit dem gemeinsam, was wir von spätägyptischen Tempelbibliotheken wissen, im Unterschied etwa zu den neuassyrischen Palastbibliotheken. Der Typus der neuassyrischen Palastbibliothek war in der Tat auf Vielfalt, Fülle und Vollständigkeit angelegt. Er war die auf die Bücherwelt Übertragene Idee des Schatzhauses. In der Tradition dieses Bibliothekstyps steht noch die Bibliothek von Alexandria und auch die heutige Institution von National-, Staats-, Landes- und Universitätsbibliotheken. Die Ägyptische Tempelbibliothek dagegen enthielt nur das Notwendige und Wichtige. ${ }^{30}$ Clemens von Alexandrien hat uns die Beschreibung einer solchen Bibliothek hinterlassen. Er spricht von 42 unabdingbaren (pany anagkaioi) Büchern, die den Grundbestand einer Tempelbibliothek ausmachten, von den Priestern in Prozessionen herumgetragen wurden und sämtlich von Thot-Hermes verfaßt sein sollten. Die Bücherprozession, von der uns Clemens Alexandrinus berichtet, gehört zu den typischen "IntellektuellenRitualen" (Bernhand Lang ${ }^{31}$ ). Die Gliederung dieses 42-er Kanons in verschiedene Abteilungen ergibt sich aus der Prozessionsordnung: ${ }^{32}$

Der Sänger trägt:

1 Buch mit Hymnen an die Götter

1 Buch mit dem Bericht über das Leben des Königs

Der Horoskopos trägt:

4 Astrologische Bücher

- über die Anordnung der Fixsterne

- über die Stellung von Sonne, Mond und den 5 Planeten

- über die Konjunktionen und Phasen von Sonne und Mond

- über die Aufgangszeiten der Sterne

30 Bibliotheken ( $p r$ md3t "Haus der Schriftrollen", sakrale Spezialbibliothek, hierá bibliothéke nach Diodor I 49.3) waren den Tempeln angegliedert und enthielten die für die Durchführung der entsprechenden Aktivitäten notwendigen Schriften, vgl. dazu G. Burkard, "Bibliotheken im alten Ägypten", in: Bibliothek, Forschung und Praxis 4. 1980. Nr.2, 79-115.

31 Berhard Lang (Hrsg.), Das tanzende Wort. Intellektuelle Rituale im Religionsvergleich, München 1984.

32 Clemens Alex., Strom. VI Cap.IV, 35.1-37, vgl. G. Fowden, The Egyptian Hermes. A Historical Approach to the Late Pagan Mind, Cambridge 1986, $58 \mathrm{f}$. 
Der Hierogrammateus trägt:

10 Hieroglyphische Bücher über Kosmographie und

Geographie, Ägypten und den Nil, Tempelbau, Landbesitz der

Tempel, Versorgung und Ausstattung der Tempel

Der Stolist trägt:

10 Bücher über Erziehung und Opferkunst, handelnd von

Weisheit und Frömmigkeit, Opferriten, Erstlingsopfer,

Hymnen, Gebete, Prozessionen und Feste

Der Prophet ${ }^{33}$ trägt:

10 Hieratische Bücher, über Gesetze, Götter und das Ganze der priesterlichen Bildung. ${ }^{34}$

Bis hierher ist die Liste klimaktisch angeordnet. Der Prophet bekleidet den höchsten, der Stolist den zweiten, der Hierogrammateus den dritten Rang usw.

Darüberhinaus gibt es nach Clemens

6 Medizinische Bücher

- über den Bau des Körpers

- über Krankheiten

- über Organe

- über Drogen

- über Augenkrankheiten

- über Frauenkrankheiten

Die überlieferten Bücherkataloge der Tempelbibliotheken von Edfu und Tod sowie gelegentliche Funde bestätigen diese Gliederung. ${ }^{35}$ Die Tendenz zur Abgrenzung und Verbindlichkeit ist in diesem Aufbau deutlich sichtbar, auch in der heiligen Zahl 42, die der Zahl der Gaue Ägyptens entspricht, sowie in Judäa die Zahl 22 bzw. 24 der Zahl der hebräischen bzw. aramäischen Buchstaben. Aus der Form und Struktur

33 Der griechische Titel prophetes überträgt den ägyptischen Titel hm-ntr "Gottesdiener" = Hohepriester, hat also nichts mit dem hebräischen Begriff des Propheten zu tun.

Der ranghöchste Priester trägt die Bücher mit dem höchsten Verbindlichkeitsgrad, vermutlich weil er als einziger zu ihrer Auslegung befugt und berufen ist.

$\mathrm{Zu}$ den Bücherkatalogen vgl. A. Grimm, "Altägyptische Tempelliteratur. Zur Gliederung und Funktion der Bücherkataloge von Edfu und et-Tod", in: SAK Beiheift 3, 1988, 168f. D.B. Redford, Pharaonic Kinglists, Annals and Daybooks: A Contribution to the Egyptian Sense of History, Mississauge 1986, 214ff. Aus Tebtunis stammen z.B. Rituale, Götterhymnen, Kosmographische und Geographische Bücher, Astromonie, Magie, Weisheitstexte, Traumbücher, Medizin, Bücher über die Tempelverwaltung, Onomastica u.a. (W.J. Tait, Papyri from Tebtunis in egyptian and in greek. London 1977; E. Reymond, From the contents of the libraries of the suchos temples in the Fayyum 2: from ancient Egyptian Hermetic Writings. Vienna 1977; Fowden, The Egyptian Hermes). 
eines Kanons ist alle Beliebigkeit ausgeschlossen. Die 42 Gaue und die 22 bzw. 24 Buchstaben sind Symbole der Ganzheit, Weltformeln, wenn man so will. Indem der Kanon diese Weltformel verwirklicht, wird er zur Welt in Buchform. ${ }^{36}$

Die hebräische Bibel zeigt alle Züge einer solchen "hochnotwendigen" Hand- und Arbeitsbibliothek. Sie ist viel eher eine Bibliothek als ein Buch. In der kanonischen Endgestalt beschränkt sie sich auf drei Abteilungen: Torah, Propheten und Schriften (in antiklimaktischer Folge). Man hat den Eindruck, da $ß$ sich mit der hebräischen Bibel die Bibliothek einer Textgemeinschaft gegen die Bibliotheken anderer Textgemeinschaften durchgesetzt hat. Diese Textgemeinschaften muß man sich nach Art der überlieferten Gruppierungen wie Sadduzäer, Pharisäer, Essener vorstellen. Zwar wird der ursprüngliche Umfang der Bibliothek von Qumran ${ }^{37}$ und der von Tebtunis auf ungefähr 1000 Schriftrollen geschätzt, was gewaltig anmutet, aber in inhaltlicher Hinsicht ist auch hier der Charakter der Arbeits- im Gegensatz zur Sammelbibliothek ziemlich deutlich.

Das gemeinsame Element der mittelalterlichen und der hellenistischrömischen Situation kann man vielleicht in der Verbindung von Textbesitz und Dissidenz erblicken. Ich möchte die These wagen, daß ohne normative Schriftlichkeit die Ausbildung solcher kollektiver Sonderwege und alternativer Lebensformen in Konfrontation zur allgemeinen und offiziellen Kultur nicht denkbar ist. Auch der ägyptische Tempel wird in der Spätzeit zum Gehäuse einer alternativen Lebensform, die durch Askese und Kontemplation gekennzeichnet ist. Die Priester sondern sich ab von der hellenisierten Kultur, aber auch von anderen Tempeln. Jeder Tempel entwickelt seine eigene Lehre und sogar sein eigenes Schriftsystem. Ungleich schärfer verlaufen jedoch die Konflikt- und Kontrastfronten in Judaea. Im antiken Judentum muß man zwischen inneren und äußeren Konfrontationen oder Kontrastfronten unterscheiden. Auf der einen Seite haben wir die inneren Konflikte zwischen Gruppierungen wie Hasmonäern, Sadduzäern, Pharisäern, Essenern usw., auf der anderen Seite die äußere Konfrontation zwischen Judaismos und Hellenismos (2 Makk $2.21)^{38}$ oder zwischen Israel und den Völkern, Jews and Gentiles. ${ }^{39}$

36 Darauf machte Aleida Assmann in der Diskussion dieses Vortrags auf der Tagung "Tradition" von J.C. Nyíri und G. Wunberg am IFK in Wien 9.-12.6. aufmerksam.

37 H. Stegemann, Die Essener, Qumran, Johannes der Täufer und Jesus, Freiburg, 3 1994, 121.

38 S. dazu Kippenberg, "Die jüdischen Überlieferungen als patrioi nomoi", in: R. Faber, R. Schlesier (Hg.), Restauration der Götter, Antike Religion und NeoPaganismus, Würzburg 1986, 45-60. 
Wenn sich das Judentum insgesamt als eine textual community gegen den Rest der Welt konstituiert, dann hat eine solche Aufrüstung sowohl von Schriftlichkeit als auch von Identität einen Vorlauf in der jahrhundertelangen Geschichte innerer Konfrontationen, in der die israelitischen und frühjüdischen textual communities gegeneinander standen.

Zwischen der ethnischen Identität und der religiösen Identität, d.h. zwischen "Israel" und dem wahren Israel wird scharf unterschieden. So wurden die Exodus-Überlieferungen in die Form einer Erinnerungsfigur gebracht, auf die hin alle geschichtlichen Konfrontationen, sowohl mit den wechselnden Fremdkulturen der Assyrer, Babylonier, Perser, Griechen, Römer usw. als auch mit der assimilationswilligen Mehrheit der eigenen Gruppe lesbar blieben - bis heute.

\subsection{Die sprachliche Engführung und die Abwehr der Idolatrie.}

Der 5. Punkt ist der schwierigste. Was haben "Idolatrie" und Traditionsverschriftung bzw. Kanonbildung miteinander zu tun? Die These ist, daß die Abwehr der Idolatrie, d.h. die Vertreibung des Göttlichen aus allen ikonischen und sonstigen Formen innerweltlicher Inkarnation mit Ausnahme der Schrift die Kanonbildung entscheidend gefördert hat. Um das zu verstehen, muß man sich klarmachen, was Idolatrie nicht bedeutet. Das Verbot, sich ein Bild zu machen, betrifft z.B. in keiner Weise die luxurierenden Anthropomorphismen der biblischen Texte, die Gott als Bräutigam, als König, als Vater und Richter, Hirten und Gärtner ausmalen. Solange die Bilder sprachlicher Art sind, ist nichts an ihnen auszusetzen. ${ }^{40}$ Die Sprache ist koscher. Das aber bedeutet, das nun all das in die Sprache hineingenommen wird, was andere Völker in einem ganzen Spektrum kultureller Ausdrucksformen entfalten. Die schriftlich fixierte Sprache ersetzt jetzt nicht nur den König, sondern auch den Tempel. Der Kanon verwandelt den Tempel in Schrift. In den Synagogen vertritt, bis heute für jeden unmittelbar evident, die Torah-Rolle das Kultbild, das wie im alten Ägypten im verschlossenen Schrein ruht, bis es den Augen enthüllt wird. Ebenso wird die Form der meditativ-mystischen Betrachtung, die "Kontemplation", vom Kultbild auf die Schrift übertragen. Die meditative oder kontemplative Lektüre entgrenzt den Sinnhaushalt des

39 S. hierzu C. Colpe, "Die Ausbildung des Heidenbegriffs von Israel zur Apologetik und das Zweideutigwerden des Christentums", in: Faber/Schlesier, Restauration, 61-87.

Vgl. Moshe Halbertal, Avishai Margalit, Idolatry, Cambridge HUP 1992, 37-66. 
Textes und führt zu einem unerschöpflichen Reichtum möglicher Auslegungen. Der "sprachlichen Engführung" korrespondiert die hermeneutische Ausweitung, die mit der "kontemplativen" Stillstellung des lesenden Blicks einhergeht.

In letzter Konsequenz ersetzt die kanonisierte Schrift die Kunst, das öffentliche Leben, tendenziell die Welt. Die Welt wird als solche zum Gegenstand der Idolatrie erklärt und diskreditiert. Die sich an den Schöpfer wendende Anbetung darf sich nicht im Geschaffenen verfangen. Der radikalen Außerweltlichkeit Gottes entspricht die radikale Schriftlichkeit seiner Offenbarung. Diesen Schritt hat das Christentum mit seiner Inkarnationstheologie rückgängig gemacht und damit den Weg zu den Bildern, zur Welt, zum Buch der Natur und letztlich auch - trotz dem in diesem Punkt eher jüdisch denkenden Augustin - zur Naturwissenschaft freigehalten. So gesehen erweist sich das Idolatrieverbot als die radikalste aller Exkarnationen.

Idolatrie entwickelt sich im Hellenismus und in der Spätantike zu dem zentralen und definierenden religiösen Abscheu des Judentums. Aus der ursprünglich politischen Kategorie der Apostasie, des Abfalls zu fremden Göttern, wird jetzt die Verteufelung von Weltbeheimatung überhaupt. Die Welt verdient kein Interesse. Alles Interesse gehört der Schrift. "Mit fünf Jahren zur Bibel, mit zehn zur Mischna, mit dreizehn zur Gebotsbeobachtung, mit fünfzehn zum Talmud, mit achtzehn ins Brautgemach, mit zwanzig zum Erwerbsleben", so lautet im Traktat Pirqe Abot der Mischna das jüdische Curriculum.

Idolatrie als kultureller Abscheu vermag am besten zu erklären, warum Ägypten trotz mancher Parallelen zum Judentum in der Antike einen anderen Weg, und letztlich unterging. Ägypten hat diesen Schritt in die Schrift nicht vollzogen, weil es an der symbolischen Präsenz des Göttlichen in der Welt festhielt. Im Horizont dieser vielfältigen kosmischen und kultischen Repräsentation ist die Sprache nur eines unter vielen Medien der Gottesnähe. In Ägypten hat sich das Göttliche nicht aus der Welt, den Bildern und Riten in die Schrift zurückgezogen. Deshalb konnte es auch nicht in der Schrift überdauern und ging zusammen mit den Bildern und Riten unter.

In der ikonoklastischen Engführung, die alles auf die Schrift konzentriert, setzt sich ein monopolistischer Zug fort, der schon die josianische Reform kennzeichnete. Ein Gott, ein Volk, ein Buch, ein Tempel, ein Medium ("keine Gestalt saht ihr, nur eine Stimme habt ihr gehört"). Dieser Weg ist wohl der am spezifischsten jüdische. Aber er hat eine Parallele im sprachlichen Ikonoklasmus der beginnenden Wissenschaft im 17.Jh., in den Versuchen, alle Bildlichkeit aus der 
Sprache zu verbannen und sie $\mathrm{zu}$ einem eindeutigen Medium wissenschaftlicher Verständigung zu machen.

Ich hoffe, es ist deutlich geworden, daß alle 5 Wege zum Kanon, die im Judentum zusammengekommen sind, typische Phänomene darstellen, die auch anderswo zu beobachten sind und die daher zum theoretischen Apparat einer historischen Phänomenologie der Schriftlichkeit gehören. Das gilt nicht nur für die Wege zum Kanon, d.h. die äußeren historischen Umstände oder "kanonogenen Situationen", sondern auch die inneren Funktionen und Auswirkungen der Kanonisierung. Davon war in meinem Beitrag überhaupt nicht die Rede. Daher möchte ich mit einem Beispiel schließen, das diesen Aspekt der Kanonisierung beleuchtet. Der Kanon erzeugt Gleichzeitigkeit. Er schafft die Illusion einer zeitlosen Gesprächssituation, in der wir mit Homer und Aristoteles kommunizieren können. Der Kanon ist anachronistisch, er ist ein Ort eigener Zeitlichkeit, ein "Chronotop" im Sinne Mikhail Bakhtins. ${ }^{41}$ Im kanonischen Chronotop sind wir Zeitgenossen Homers und Platons, Moses und Jesaias. Ich möchte das mit einer vielzitierten, inzwischen schon geradezu abgedroschenen Geschichte aus dem Talmud illustrieren, die ich nach Yerushalmi zitiere:

"In der Stunde, da Mose zur Höhe aufstieg [um die Tora in Empfang zu nehmen], fand er den Heiligen, gelobt sei er, wie er dasaß und taggin [kronenähnliche Schnörkel] anknüpfte. Er sagte vor ihm: Herr der Welt, wer hindert deine Hand? [D.h. fehlt in der Tora etwas, so daß derlei Ornamente nötig wären?] Er sprach zu ihm: Es ist ein Mensch, der zukünftig, am Ende vieler Generationen sein wird - Akiba, Josephs Sohn ist sein Name - der zukünftig über jedes einzelne Strichlein ganze Berge von Lebensregeln auslegen wird. Er sagte zu ihm: Herr der Welt! Laß mich ihn sehen. Er sprach zu ihm: Wende dich nach hinten! Moses ging [in Rabbi Akibas Akademie] und setzte sich am Schluß von acht Reihen hin. Er verstand aber nicht was sie sagten. Da verlor er seine Fassung. Als er zu einer bestimmten Sache kam, da sagten seine Schüler zu ihm: Meister, woher hast du das? Er sagte zu ihnen: Es ist eine Lebensregel an Mose vom Sinai. Da beruhigte sich sein Sinn".42

Mose im Bet Midrasch von Rabbi Akiba: das ist Nietzsches Geistergespräch more talmudico, die Stillstellung und Verräumlichung der Zeit in einer kanonisierten Tradition.

41 Mikhail Bakhtin, The Dialogic Imagination, hg. u. übers. v. Caryl Emerson und Michael Holquist, Austin 1981, 84.

42 bMenachot 29b, nach Y.H. Yerushalmi, Zakhor. Erinnere Dich! Jüdische Geschichte und Jüdisches Gedächtnis, Berlin 1988, 32. 